Cite this: Soft Matter, 2013, 9, 3465

Received 7th December 2012

Accepted 30th January 2013

DOI: $10.1039 / \mathrm{c} 3 \mathrm{sm} 27809 \mathrm{c}$

www.rsc.org/softmatter

\title{
Stimuli-responsive hydrogels cross-linked by magnetic nanoparticles
}

\author{
Patrick $\| \mathrm{g}$ * \\ Embedding magnetic nanoparticles into soft host media offers the opportunity to externally control \\ material properties via a magnetic field. Choosing a hydrogel as the host medium allows modification \\ of not only the elastic properties, but also the degree of swelling of the gel and the shape changes of \\ the sample. Hydrogels where magnetic nanoparticles serve as the only crosslinking reagent of the \\ network are a promising new class of such stimuli-responsive gels. The well-defined magneto- \\ mechanical coupling present in these materials should allow for a better understanding and \\ optimization of field-induced changes.
}

\section{Introduction}

Stimuli-responsive soft matter systems constitute a fascinating class of materials with promising applications in various fields. Soft magnetic materials like magneto-rheological fluids provide an example of materials in which the mechanical yield stress can be tuned by varying an external magnetic field. ${ }^{1}$ In the last few years, magnetic gels have been synthesized ${ }^{2-5}$ where magnetic particles are embedded into an elastic matrix. In these materials, the degree of elasticity changes with external magnetic field strength. ${ }^{6}$ In order to achieve significant changes in elastic moduli, the host medium should be rather soft which, however, often allows nanoparticles to diffuse out of the gel. Recent advances in synthesis have shown that a good solution to this problem can be found, with a strong hardening of the material in the presence of a magnetic field. ${ }^{7,8}$ For a recent review, see ref. 9.

In the last few years, these more traditional magnetic gels and elastomers have been complemented by hydrogels

ETH Zürich, Department of Materials, Polymer Physics, HCI H529, CH-8093 Zürich, Switzerland

Patrick Ilg received his Master's degree in Physics from the University of Göttingen, Germany, in 1997. He obtained his Ph.D. degree in Physics from the ETH Zürich, Switzerland, in 2001. After working as a post-doc at the Technical University Berlin, Germany, and the Universite Claude Bernard in Lyon, France, he received his habilitation in Theoretical Physics from the TU Berlin in 2006. Also since 2006, he has been a senior researcher at the Department of Materials at ETH Zürich, Switzerland. His research interest comprises the structure and dynamics of soft and amorphous matter as well as systematic scale-bridging methods. containing magnetic nanoparticles. Hydrogels are cross-linked networks formed by hydrophilic polymers. A characteristic feature of hydrogels is their enormous swelling in aqueous media. Contrary to elastomers, hydrogels are very soft materials. Their mechanical properties, however, can be changed by incorporating nanoparticles into the gel. ${ }^{10}$ Magnetic hydrogels, where magnetic nanoparticles are incorporated into the hydrogel, could provide a fascinating opportunity to reversibly change their mechanical properties by an external magnetic field. For the corresponding case of magnetic nanoparticles in a non-polar solvent ("ferrofluid"), the field-dependence of the effective viscosity is well-established. ${ }^{11}$ In hydrogels with micron-sized magnetic particles, strong field-dependent changes of the shear modulus ${ }^{12}$ and sample shape ${ }^{13}$ have been observed. Due to their small size, magnetic nanoparticles have a permanent dipole moment and tend to diffuse much faster compared to their micro-sized counterparts. ${ }^{14}$ When suspended in a non-polar solvent, magnetic nanoparticles typically show superparamagnetic behavior. In hydrogels, magnetic nanoparticles are prone to show magnetic hysteresis $^{15}$ since particle rotation is severely hindered in the polymer matrix and Néel relaxation within the particles is slow. Also the interesting phenomenon of self-healing has been observed in a hydrogel doped with magnetic nanoparticles. ${ }^{\mathbf{1 6}}$ In addition, thermoreversible ferrohydrogels are available now, where the type and strength of the gel can be modified by temperature changes. ${ }^{17}$ Strong but reversible shape changes of a magnetic hydrogel observed upon changing the external magnetic field strength have been found in ref. 18. Such an effect can be very useful not only for artificial muscles but also for several other applications such as microfluidic valves, ${ }^{\mathbf{1 9}}$ actuators, ${ }^{20}$ contrast agents in magnetic resonance, ${ }^{21}$ hyperthermia ${ }^{22}$ or controlled drug delivery. ${ }^{23,24}$ For a recent review on diverse biomedical applications of magnetic hydrogels see ref. 25. 


\section{Magnetically linked hydrogels}

A new type of hydrogel with magnetic nanoparticles as the crosslinking reagent has been presented in ref. 26-28. In these works, the innovative method of producing a magnetic hydrogel is to avoid the use of additional cross-linkers in the polymerization step and to use instead the nanoparticles themselves as the only crosslinking reagents. In order to achieve this, the surfaces of the nanoparticles have to be functionalized appropriately. The same magnetic material $\left(\mathrm{CoFe}_{2} \mathrm{O}_{4}\right)$ with a comparable size of the individual nanoparticles of around $10 \mathrm{~nm}$ has been used in all three studies. ${ }^{26-28} \mathrm{CoFe}_{2} \mathrm{O}_{4}$ nanoparticles of this size are large enough that the magnetic moment is effectively blocked within the particle. Yet the nanoparticles are still small enough so that they can be considered a magnetic monodomain. The magnetic nanoparticles are combined with different polymers and correspondingly different functional groups on their surfaces. The hydrogels are based on polyethylene glycol (PEG) and acrylamide, ${ }^{26}$ poly(acrylamide) (PAAm),${ }^{27}$ and polysaccharide, ${ }^{28}$ respectively. In either case, the functionalized magnetic nanoparticles form covalent bonds with the polymer network.

The advantage of such an approach is that the magnetic nanoparticles are permanently linked to the network which prevents them from diffusing out of the gel. A loss of magnetic material would degrade the properties of the hydrogel or even lead to toxic effects in biomedical applications. Using the magnetic nanoparticles as crosslinking reagents instead of attaching them somewhere on the strands of the network opens the possibility to study the magneto-mechanical coupling in more detail. While clustering or some sort of structure formation is considered to be essential for significant field-induced changes in other systems, ${ }^{6,7}$ these gels in principle allow transmission of torques due to an external magnetic field onto the polymer network via the rotation of individual magnetic nanoparticles. Such a well-defined magneto-mechanical coupling could allow for more refined theoretical approaches, leading to a better understanding of these systems and consequently an improved control of field-induced changes in material properties. To this end, the ferrohydrogels need to be well-characterized. A number of their properties have been established by now. First, the magnetic gels show the characteristic equilibrium swelling behavior of hydrogels. ${ }^{27}$ Next, the hydrogels are found to remain stable even after many swelling/washing cycles, indicating a chemically cross-linked network. ${ }^{27,28}$ Also, since no magnetic material was detected during the washing step, it is safe to assume that the magnetic nanoparticles are indeed permanently linked to the polymer network. Fig. 1 shows that the magnetic hydroge ${ }^{27}$ remains stable even after the magnetic cores of the nanoparticles have been dissolved, as long as the functionalized shell remains intact.

The detailed internal organization of these magnetic hydrogels, however, has not been fully clarified yet. Rheological tests show a noticeable change of the yield stress with waiting time after preparation, indicating the presence of slow relaxation or reorganization processes in these hydrogels. ${ }^{28}$ SEM and AFM measurements show that the nanoparticles lead to an (a)

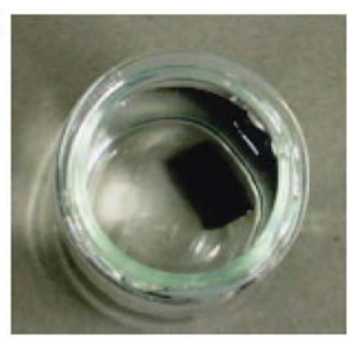

(b)

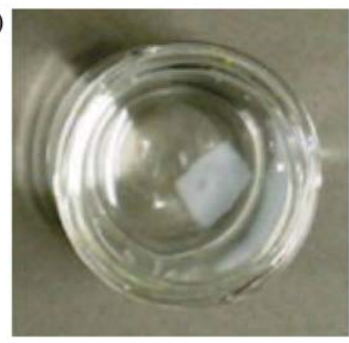

(c)

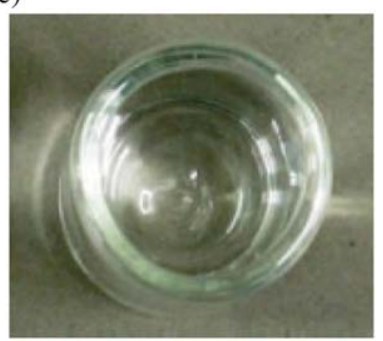

Fig. 1 (a) Photograph of the swollen magnetic hydrogel. (b) Hydrogel after the magnetic cores have been dissolved. (c) Polymer solution after the hydrogel has been degraded. Reprinted with permission from [27]. Copyright 2011 American Chemical Society.

increased surface roughness on the order of $50 \mathrm{~nm} .{ }^{28}$ From comparisons of TEM image analysis, X-ray diffraction experiments and additional magnetization measurements of waterbased dispersions of the nanoparticles, a comparable mean core diameter can be determined, suggesting that no significant particle agglomeration occurs. ${ }^{27}$ Furthermore, TEM image analysis and X-ray tomography show that the magnetic nanoparticles are well-dispersed and that the hydrogel obtained in ref. 27 is spatially homogeneous. A very rough estimate of the typical mesh size of the swollen hydrogel from TEM image
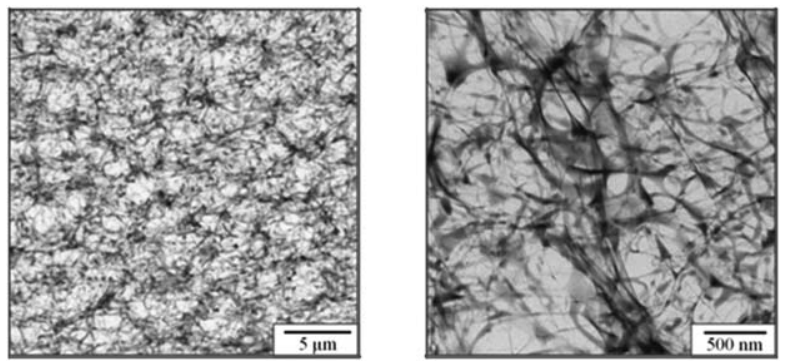

Fig. 2 TEM images of the magnetic hydrogel at low (left) and high (right) resolution. Reprinted with permission from [27]. Copyright 2011 American Chemical Society. 
analysis (see Fig. 2) gives a value of the order $150 \mathrm{~nm}$, in good agreement with the mean distance between nanoparticles. ${ }^{27}$ The mesh size is a very important parameter of hydrogels in general as it controls, e.g., the degree of swelling. The mesh size itself is controlled by the density of crosslinking reagents which in this case are the magnetic nanoparticles. Indeed, the concentration of magnetic nanoparticles is found to control the degree of swelling: with increasing nanoparticle concentration the mean distance between the network nodes shrinks and the hydrogel is less able to swell. ${ }^{27}$

The successful preparation of a related but more complicated magnetic hydrogel has been reported in ref. 29. A TEM image of this hydrogel is shown in Fig. 3. In this hierarchically structured gel, not only the magnetic nanoparticles but also liquid-crystalline domains that are formed by amphiphilic block-copolymers serve as crosslinking reagents. This hydrogel is physically cross-linked by noncovalent bonds. Nevertheless, the resulting ferrogel shows good mechanical stability, similar to that of chemically cross-linked hydrogels. Since the ferrogel is free-standing and bending in an external field gradient, the material could be used as a soft actuator or artificial muscle.

The details of the network architecture obtained in the magnetic hydrogels ${ }^{26-29}$ are not yet fully known. Also, the magnetic field-dependence of this new type of ferrohydrogels has not been carefully explored to date. Since the crosslinking density in these hydrogels is related to the concentration of magnetic nanoparticles, varying the latter not only changes the mechanical properties, mesh size, biodegradation, etc. but also the magnetic properties. These interesting aspects certainly deserve further studies. Theoretical works on the field-induced deformation of ferrogels and ferroelastomers (magnetostriction) are available in the literature, predicting elongation or

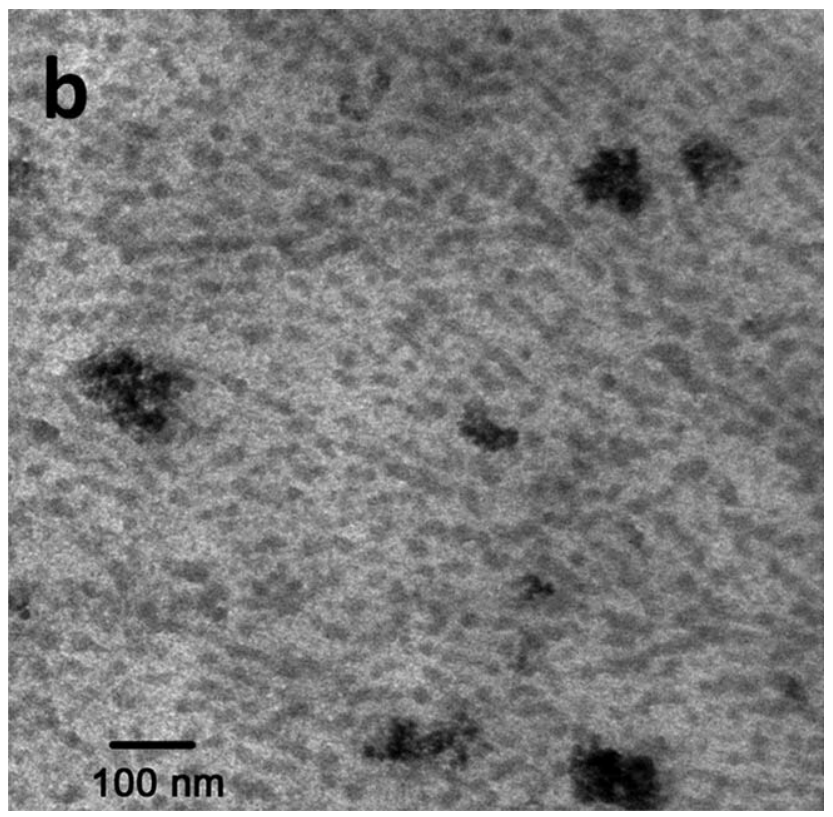

Fig. 3 TEM image of the hierarchically structured hydrogel containing magnetic nanoparticles as well as liquid-crystalline domains formed by block copolymers. A few large-size clusters (around $200 \mathrm{~nm}$ ) of magnetic nanoparticles are present. Reprinted with permission from [29]. Copyright 2012 American Chemical Society.
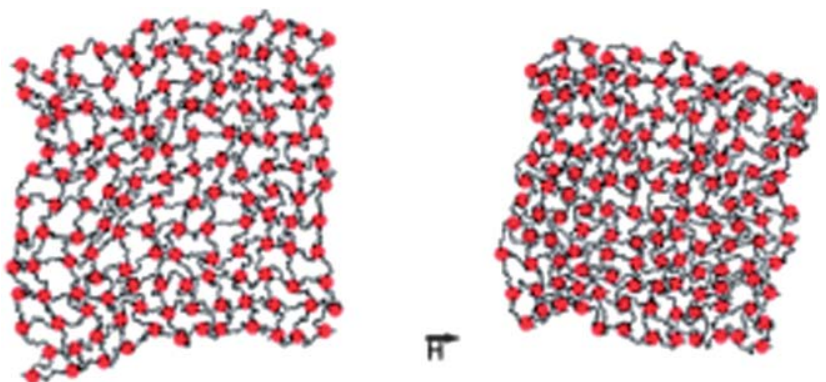

Fig. 4 Snapshot from computer simulations of a bead-spring network model with embedded magnetic particles without (left) and with (right) an applied magnetic field. From ref. 33

contraction of the gel along the field direction depending on the initial shape of the sample, field strength and particle concentration. $^{30-32}$ As macroscopic, continuum approaches, those theories do not take into account that the magnetic nanoparticles also serve as crosslinking reagents in this new type of hydrogel. A first simulation work of such a magnetic gel has already appeared. ${ }^{33}$ The model studied there considers an idealized situation, where the polymer strands and magnetic nanoparticles are linked by spring forces, initially forming a regular array. For simplicity, the simulations are restricted to two dimensions. A snapshot of the simulations is shown in Fig. 4. The dipole moments of the magnetic particles experience a torque due to an external field. In the model, this torque is transmitted to the network via the spring forces that are attached to the magnetic particles. This model indeed shows a certain degree of magnetic field-induced shape change. Further analysis of the deformations and comparisons with the corresponding ones in more traditional hydrogels ${ }^{34}$ would certainly be interesting.

\section{Conclusions}

Magnetic gels are a fascinating class of stimuli responsive materials. How their properties change in a magnetic field and how to control and optimize those effects are important questions to address before such materials can be used in practical applications. So far, however, these questions were very difficult to answer and progress was slow, since the precise coupling of the magnetic nanoparticles to the gel matrix was not wellknown.

The situation has changed substantially with the new class of magnetic hydrogels presented in ref. 26-28. Since magnetic nanoparticles form the nodes of the polymer network, torques on the particles due to a magnetic field are transmitted to the connected polymer strands. Such a well-defined coupling opens the way for a better understanding of the magneto-mechanical properties and subsequent synthesis of magnetic hydrogels with improved properties. Having identified the physical mechanism of torque transmission from the magnetic field to the gel, theory and simulation are in a good position to contribute to the optimization and improved control of such magnetic gels. First simulation work in this direction has already appeared where a simplified bead-spring network 
model was studied. ${ }^{33}$ There will hopefully be a number of subsequent studies that explore the field-dependent properties and deformations of such hydrogels.

\section{Acknowledgements}

Financial support from the Swiss National Science foundation SNSF (grant no. 20PA21E-129506) is gratefully acknowledged.

\section{References}

1 J. da Vicente, D. J. Klingenberg and R. Hildago-Alvarez, Soft Matter, 2011, 7, 3701.

2 M. Zrínyi, Trends Polym. Sci., 1997, 5, 280.

3 Z. Varga, G. Filipcsei, A. Szilágyi and M. Zrínyi, Macromol. Symp., 2005, 227, 123-134.

4 M. Zrínyi, Colloid Polym. Sci., 2000, 278, 98-103.

5 D. Collin, G. K. Auernhammer, O. Gavat, P. Martinoty and H. R. Brand, Macromol. Rapid Commun., 2003, 24, 737-741. 6 Z. Varga, G. Filipcsei and M. Zrínyi, Polymer, 2006, 47, 227-233. 7 H. An, S. J. Picken and E. Mendes, Soft Matter, 2010, 6, 44974503.

8 T. Mitsumata, S. Ohori, A. Honda and M. Kawai, Soft Matter, 2013, 9, 904-912.

9 H. R. Brand, P. Martinoty and H. Pleiner, in Cross-linked Liquid Crystalline Systems: From Rigid Polymer Networks to Elastomers, ed. G. Crawford, D. Broer and S. Zumer, Taylor and Francis, 2011, pp. 529-563.

10 R. Messing and A. M. Schmidt, Polym. Chem., 2011, 2, 18-32. 11 S. Odenbach, J. Phys.: Condens. Matter, 2003, 15, S1497-S1508.

12 J. Wu, X. Gong, Y. Fan and H. Xia, Soft Matter, 2011, 7, 62056212.

13 R. V. Ramanujan and L. L. Lao, Smart Mater. Struct., 2006, 15, 952.

14 Magnetic nanoparticles, ed. S. P. Gubin, Wiley-VCH, Berlin, 2009.

15 S. Monz, A. Tschöpe and R. Birringer, Phys. Rev. E: Stat. Phys., Plasmas, Fluids, Relat. Interdiscip. Top., 2008, 78, 021404.

16 Y. Zhang, B. Yang, X. Zhang, L. Xu, L. Tao, S. Li and Y. Wei, Chem. Commun., 2012, 48, 9305-9307.
17 M. Krekhova, T. Lang, R. Richter and M. Schmalz, Langmuir, 2010, 26, 19181-19190.

18 R. Fuhrer, E. K. Athanassiou, N. A. Luechinger and W. J. Stark, Small, 2009, 5, 383-388.

19 N. S. Satarkar, R. E. E. W. Zhang and J. Z. Hilt, Lab Chip, 2009, 9, 1773-1779.

20 V. Q. N. R. L. Snyder and R. V. Ramanujan, Smart Mater. Struct., 2010, 19, 055017.

21 J. I. Kim, C. Chun, B. Kim, J. M. Hong, J. K. Cho, S. H. Lee and S. C. Song, Biomaterials, 2012, 33, 218-224.

22 S. A. Meenach, J. Z. Hilt and K. W. Anderson, Acta Biomater., 2010, 6, 1039-1046.

23 A. T. Paulino, A. G. B. Pereira, A. R. Fajardo, K. Erickson, M. J. Kipper, E. C. Muniz, L. A. Belfiore and E. B. Tambourgi, Carbohydr. Polym., 2012, 90, 1216-1225.

24 G. Giani, S. Fedi and R. Barbucci, Polymers, 2012, 4, 11571169.

25 Y. Li, G. Huang, X. Zhang, D. Li, Y. Chen, T. Lu, T. J. Lu and F. Xu, Adv. Funct. Mater., 2012, DOI: 10.1002/ adfm.201201708.

26 M. Bonini, S. Lenz, R. Giorgi and P. Baglioni, Langmuir, 2007, 23, 8681-8685.

27 R. Messing, N. Frickel, L. Belkoura, R. Strey, H. Rahn, S. Odenbach and A. M. Schmidt, Macromolecules, 2011, 44, 2990-2999.

28 R. Barbucci, D. Pasqui, G. Giani, M. D. Cagna, M. Fini, R. Giardino and A. Atrei, Soft Matter, 2011, 7, 55585565.

29 Y. Zhou, N. Sharma, P. Deshmukh, R. K. Lakhman, M. Jain and R. M. Kasi, J. Am. Chem. Soc., 2012, 134, 1630-1641.

30 E. Jarkova, H. Pleiner, H.-W. Müller and H. Brand, Phys. Rev. E: Stat. Phys., Plasmas, Fluids, Relat. Interdiscip. Top., 2003, 68, 041706.

31 S. Kankanala and N. Triantafyllidis, J. Mech. Phys. Solids, 2004, 52, 2869-2908.

32 A. Y. Zubarev, Soft Matter, 2012, 8, 3174-3179.

33 G. Weeber, S. Kantorovich and C. Holm, Soft Matter, 2012, 8, 9923-9932.

34 Q. Wen, A. Basu, P. A. Janmey and A. G. Yodh, Soft Matter, 2012, 8, 8039-8049. 